\title{
WAVELET FUSION FOR CONCEALED OBJECT DETECTION USING PASSIVE MILLIMETER WAVE SEQUENCE IMAGES
}

\author{
Yang Chen, Lei Pang, Hui Liu, Xigui Xu \\ Beijing University of Civil Engineering and Architecture \\ No.15 ,yongyuanlu Road, DaXing District, 102600, Beijing, China
}

\section{Commission VI, WG III/2}

KEY WORDS: passive millimeter wave, sequence images, sum of squared difference, wavelet fusion, concealed object detection

\begin{abstract}
:
PMMW imaging system can create interpretable imagery on the objects concealed under clothing, which gives the great advantage to the security check system. Paper addresses wavelet fusion to detect concealed objects using passive millimeter wave (PMMW) sequence images. According to PMMW real-time imager acquired image characteristics and storage methods, firstly, using the sum of squared difference (SSD) as the image-related parameters to screen the sequence images. Secondly, the selected images are optimized using wavelet fusion algorithm. Finally, the concealed objects are detected by mean filter, threshold segmentation and edge detection. The experimental results show that this method improves the detection effect of concealed objects by selecting the most relevant images from PMMW sequence images and using wavelet fusion to enhance the information of the concealed objects. The method can be effectively applied to human body concealed object detection in millimeter wave video.
\end{abstract}

\section{INTRODUCTION}

Passive millimeter wave (PMMW) imaging systems image by detecting differences in ambient and target radiant temperatures in a scene. It does not need to launch electromagnetic waves, so it will not cause radiation damage to the human body (Kemp M C, 2008). Millimeter waves have a certain ability to penetrate, so it can be used to detect objects hidden under clothing. The application of millimeter wave to security areas can enrich the security approach, strengthen security capabilities and rate, improve the security system. At present, the common PMMW security checking system needs the cooperation of the

Corresponding author: panglei@ bucea.edu.cn(Lei Pang) testers to collect static single-frame images for concealed object detection (For example, Brijot's GEN2 system). However, the limited information provided by single-frame images cannot meet the demand of security inspection of the diversity of concealed objects in many cases (Song et al, 2011; Zhang et al, 2015).

In order to meet the diversified requirement of security checking, researchers have successfully developed the array passive millimeter wave real-time imaging system (Miao et al, 2013). This imaging system enables fast, real-time human imaging, and it greatly improves the imaging rate and rich information, but also poses some challenges to the hidden object detection algorithm. How to rapidly and 
accurately detect human-body concealed objects from a large number of acquired sequence images has become an urgent problem to be solved. Now, there are few detection algorithms for concealed objects in sequence images by researchers both at home and abroad. Most detection algorithms are aimed at single-frame PMMW images with low signal-to-noise ratio, low spatial resolution and low contrast.

Among them, some researchers have successfully applied the expectation maximization and hierarchical segmentation methods to detect occult objects, such methods mainly through segmenting effectively the background, human body and goal of the PMMW image (Lee et al, 2009; Yeom et al, 2010). In addition, some researchers use the morphological filtering and wavelet denoises to improve the quality of the PMMW image to better detect the concealed objects (Qin et al, 2012). And, some researchers also choose to use three iterations to enhance the image quality first, then the global threshold is used to segment the human body and the background, and finally, the human body and the target are segmented by the second iterative method to detect the concealed object (Mu et al, 2014). The successful implementation of these algorithms is helpful to the research of detection methods of human-body concealed object detection of PMMW sequence images.

The paper is organized as follows. In Section 2, we introduce the passive millimeter wave imaging principle, SSD and wavelet fusion. The detection algorithm of human-body concealed object of PMMW sequence images is discussed in Section3. In Section 4, we present the experimental results with the performance evaluation. The conclusions follow in Section 5 .

\section{BASIC PRINCIPLE}

\subsection{The PMMW Imaging Principle}

PMMW imaging system images by detecting the difference in millimeter-wave energy of the target radiation. As illustrated in Figure 1, passive millimeter wave imaging system mainly includes: antenna, radiometer receiver, servo control device, signal processor and display control device.

Passive millimeter wave imaging system at work, the antenna receives the radiation of the target millimeter wave radiation energy. Millimeter wave radiation brightness of the target is converted to the corresponding low-frequency components via Radiometer receiver, and then the radiometer output signal is real-time sampling and recording by the microcontroller. Each recorded gray-scale pixel corresponds to a sampling point. Finally, the control device stores and displays the corresponding millimeter wave image.

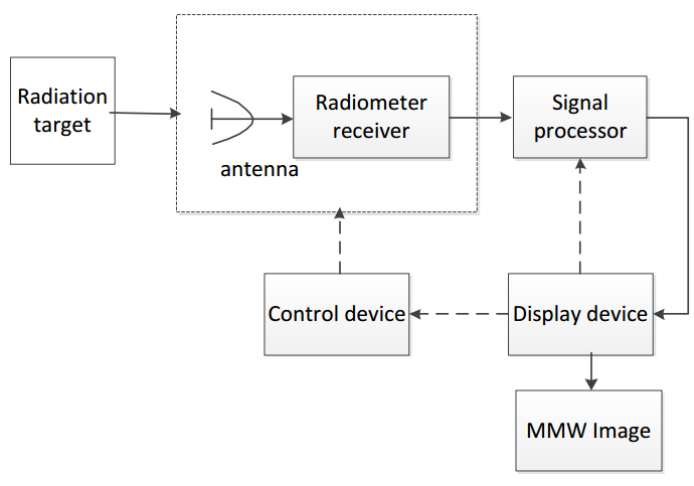

Figure 1. Schematic of passive millimeter wave imaging principle

\subsection{SSD}

The similarity of the two images can be quantitatively expressed by the SSD. The smaller the SSD, the more relevant the two images are. ( i.e. the difference between the radiation values of the corresponding elements in the two images is very little.) The specific mathematical model of SSD is as follow.

$\mathrm{R}\left(I_{i}, I_{j}\right)=\sum_{x, y}\left(I_{i}(x, y)-I_{j}(x, y)\right)^{2}\left(i, j \in \mathbf{N}^{*}\right)$

where $\quad x, y=$ coordinates of the pixel

$\mathrm{I}_{\mathrm{i}}(x, y), \mathrm{I}_{\mathrm{j}}(x, y)=$ radiation values at $(x, y)$ in the $\mathrm{i}$-th and $\mathrm{j}$-th images, respectively. $\mathrm{R}\left(\mathrm{I}_{\mathrm{i}}, \mathrm{I}_{\mathrm{j}}\right)=$ SSD between images $i$ and $j$. 


\subsection{Wavelet Fusion}

Image fusion refers to the technology that extracts information from multiple images with the same scene. Through it you can get a higher quality image (Zhang et al, 2014). Wavelet transform has perfect reconstruction ability; it can decompose the original image into a series of sub-images with different spatial resolution and frequency-domain characteristics. These sub-images fully reflect the local variation of the original image. In the process of fusion, as illustrated in Figure 2, the original image is first decomposed into a series of channels, and then the decomposed tower structure is used to fuse the features and details carried by each image in multiple decomposition layers and different frequency bands (Tao et al, 2003). The steps of wavelet fusion are as follows.

a) Wavelet decomposition is performed on each source image separately to establish wavelet pyramid decomposition of the image.

b) The decomposition layers are fused separately, and different fusion operators are used to fuse the different frequency components of each decomposition layers. Finally, the fused wavelet pyramid is obtained.

c) The fusion image can be obtained by inverse wavelet transform of the fused wavelet pyramid.

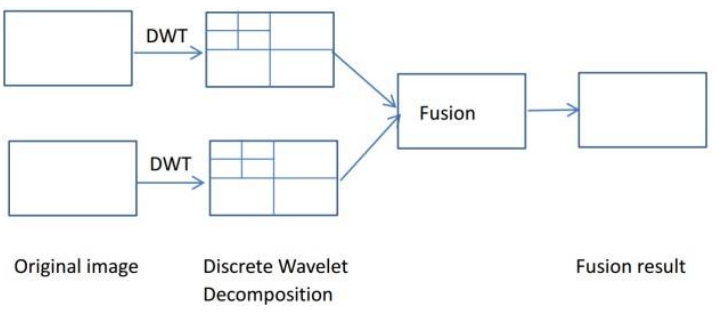

Figure 2. Schematic of wavelet fusion

\section{THE DETECTION ALGORITHM OF CONCEALED OBJECT OF PMMW SEQUENCE IMAGES}

Due to the complicated imaging environment and hardware limitations, PMMW real-time imager acquires barely single-frame images with high quality. Therefore, the detection of single-frame images may result in missed inspection or false detection of concealed objects. In response to this defect, this paper uses sequence images to enhance the amount of information, and put forward the detection methods of human-body concealed object of PMMW sequence images. Algorithm flow shown in Figure 3 and the algorithm is divided into the following steps:

I Enter $n$ frames of PMMW sequence images

II Calculate SSD between two images, and record as $\mathrm{R}\left(I_{i}, I_{j}\right), i, j \in[1, n]$

III Obtaining the index $(i, j)$ when $\operatorname{Min}\left(\left(R\left(I_{i}, I_{j}\right)\right)\right.$

IV the selected image $I_{i}$ and $I_{j}$ is processed by wavelet fusion, get the fusion image $I$

$\mathrm{V}$ Image $I$ through mean filtering, threshold segmentation, edge detection to generate detection results $I_{e}$

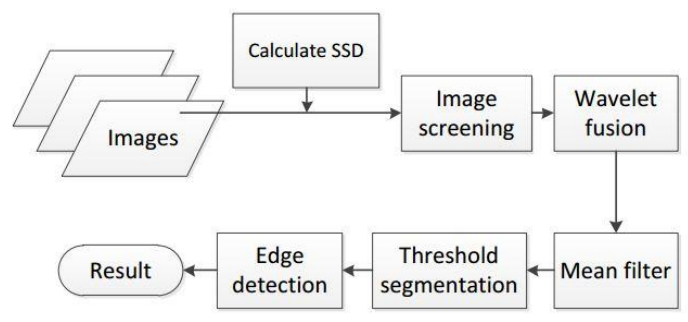

Figure 3. Flow Chart of the detection method of human-body concealed object

According to the known principle, make the following experimental steps:

a) Obtain the original image of security check: capture the simulated sequence images of security check through an 8mm-band PMMW real-time imager.

b) Image screening: according to equation (1) calculate the SSD between the sequence of images, select two images of the smallest SSD as the later detection image.

c) The fusion results are obtained by wavelet fusion of the selected images. 
d) The $3 \times 3$ mean filter algorithm is used to denoise the fused image, followed by threshold segmentation, and finally the canny operator is used to detect the edge of the concealed object.

e) Output experiment results and verify

\section{EXPERIMENTAL RESULTS AND ANALYSIS}

The sequence images of the experimental data are collected by the PMMW real-time imager. Five adjacent sequence images containing occult objects are selected as the detection data, and the hidden object detection experiment is performed by using the above-described the detection methods of human-body concealed object of PMMW sequence images.

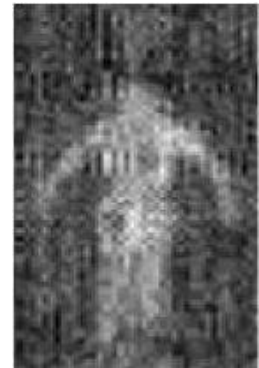

$\left(I_{1}\right)$

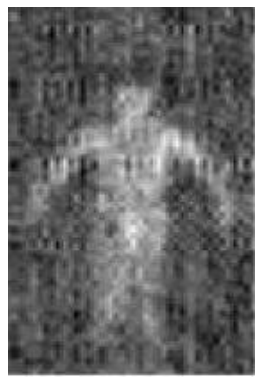

$\left(I_{3}\right)$

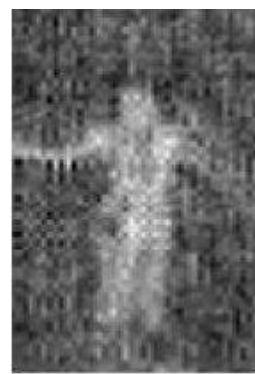

$\left(I_{5}\right)$

Figure 4. Five frame sequence images of PMMW
As shown in Figure $4, I_{1}, I_{2}, I_{3}, I_{4}$ and $I_{5}$ are five frames of PMMW sequence images with hidden objects. The bright part inside the body is the concealed object. Since the five images in Figure 4 contain a lot of noise and the content of the hidden object is small. Therefore, only the concealed object detection of a single frame image cannot obtain an effective result.

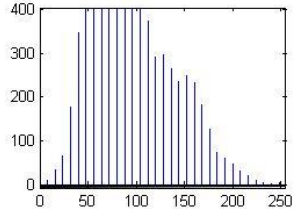

(a)

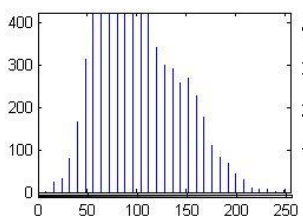

(c)

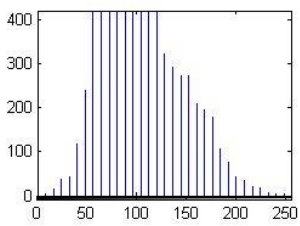

(e)

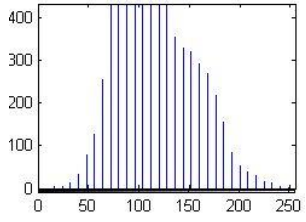

(b)

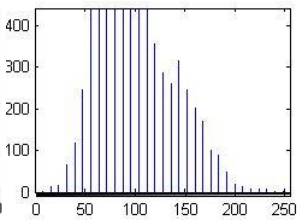

(d)

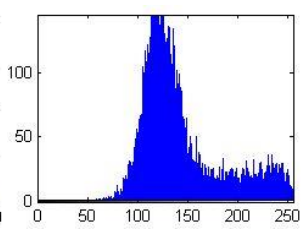

(f)
Figure 5. Figure(a), (b), (c), (d) and (e) represent the histogram of the five frame sequence images respectively, and figure(f) shows the histogram of the fused image.

Figure 5. Figure(a), (b), (c), (d) and (e) represent the histogram of the five frame sequence images respectively. Due to the scattered information in single-frame PMMW images, the information of the concealed objects cannot be directly segmented. As shown in Figure 5(f), through the relevant screening, wavelet fusion and mean filter processing these images can make the image background and hidden information highlighted. 
Optimizing and screening images with SSDs without missing the necessary information can reduce the amount of data that need detection. The bigger advantage is that the selected images can enhance the hidden information and improve the detection speed and accuracy. Screening with SSD as a parameter, the images $\mathrm{I}_{3}$ and $\mathrm{I}_{4}$ with the smallest SSD are selected as the detection images. SSD of two image $\mathrm{R}_{34}=0.38167 \times 103$, as illustrated in Table. 1 .

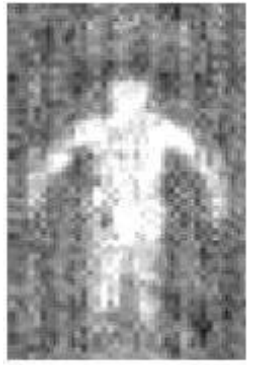

(a)

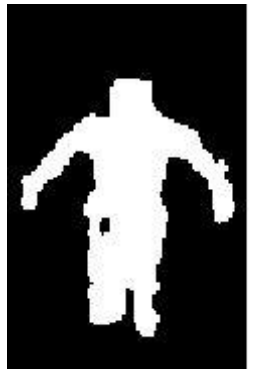

(c)

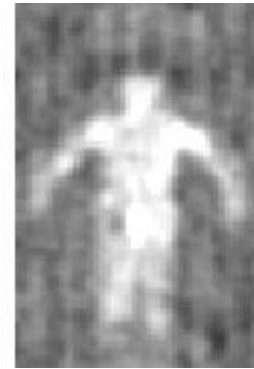

(b)

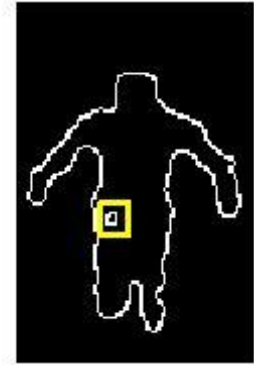

(d)
Figure 6. Figure (a), (b), (c) and (d) represent the results of wavelet fusion, mean filter, threshold segmentation and edge detection respectively.

The filtered images are processed by wavelet fusion, mean filter, threshold segmentation and edge detection. Figures 6(a), 6(b),6(c) and 5(d) shows the result of the human-body concealed object detection process. As shown in Figure 6(a), the image of wavelet fusion greatly preserves the original information of the image. However, in order to enhance the information intensity of the concealed objects, noise information is also inevitably enhanced. The $3 \times 3$ mean filter is used to smoothen the fused image, and background noise of the filtered image is obviously suppressed, as shown in Figure 6(b). Figure 6(c) shows the result of the detection of the filtered image by threshold segmentation and edge detection. The yellow box in Figure 6(d) shows the range of concealed object.

\begin{tabular}{cc}
\hline Number & SSD $\left(10^{3}\right)$ \\
\hline $\mathrm{R}_{12}$ & 7.9712 \\
$\mathrm{R}_{13}$ & 0.8605 \\
$\mathrm{R}_{14}$ & 1.047 \\
$\mathrm{R}_{15}$ & 1.7528 \\
$\mathrm{R}_{23}$ & 4.3382 \\
$\mathrm{R}_{24}$ & 4.5896 \\
$\mathrm{R}_{25}$ & 2.8134 \\
$\mathrm{R}_{34}$ & 0.38167 \\
$\mathrm{R}_{35}$ & 0.53081 \\
$\mathrm{R}_{45}$ & 0.64395 \\
\hline
\end{tabular}

Table 1 Related parameters SSD of arbitrary two images in PMMW sequence images.

\section{CONCLUSIONS}

In this paper, we have proposed a new approach to PMMW sequence images. The PMMW images usually have the low contrast and resolution. Also, the images are often noisy due to the low-signal level. Therefore, we have presented a special method that combination of SSD and wavelet fusion to detect human body concealed objects in PMMW sequence images. In sum up, the detection algorithm of human-body concealed objects in PMMW sequence images makes up for the shortcomings of the lack of single frame image information. And it enhances the ability to detect hidden objects by optimizing screening and fusion of important information in sequence images.

In the future, the rapid detection and identification of hidden objects in millimeter-wave video will be focus of the next research.

\section{ACKNOWLEDGMENTS}

We are grateful for the experimental data provided and support by the School of Electronic and Information Engineering of Beihang University. 


\section{REFRENCES}

Kemp M C, 2008. Millimeter wave and terahertz technology for detection of concealed threats - a review// Joint, International Conference on Infrared and Millimeter Waves, 2007 and the 2007, International Conference on Terahertz Electronics. Irmmw-Thz. IEEE, pp.:647-648.

Lee D S, Yeom S, Son J Y, et al, 2009. Image segmentation of concealed objects detected by passive millimeter wave imaging, International Conference on Infrared, Millimeter, and Terahertz Waves. IEEE, pp.:1-2.

Miao J, Zhang C, Hu A, et.al, 2013. Real-time Passive Millimeter Wave Imaging Technology, Journal of Microwaves. Vol.29 (z1), pp.:100-112.

Mu S, Shan H, Zhou J, et.al, 2014. A Method for Detecting Human Concealed Objects in Passive Millimeter Wave Image, Technology Information Journal, pp.:202-203.

Qin W, Zhang G and Lou G, 2012. Feature extraction method of PMMW radiation image based on security inspection, Computer Engineering and Applications, Vol. 48(28), pp.:193-196.

Song S, Wang X and Deng J, 2011. Status and the Key Techniques of Passive Millimeter-Wave Imaging System, SCENCE \& TECHNOLOGY REVIEW. Vol.29 (19), pp.:74-79.

Tao G, Li D and Lu G, 2003. Study on image fusion based on different fusion rules of wavelet transform, Infrared and Laser Engineering, Vol. 32(2), pp.:173-176.

Yeom S, Lee D S, Son J Y, et al, 2010. Concealed object detection using passive millimeter wave imaging, Universal Communication Symposium. IEEE, pp.:383-386.

Zhang H, Hong W, Chen P, et.al, 2015. Novel microwave imaging system based on direct digital synthesizer, Chinese Journal of Radio Science, Vol.30 (4), PP.:704-708

Zhang $\mathrm{X}, \mathrm{Li} \mathrm{X}$ and $\mathrm{Li} \mathrm{J}$, 2014. Validation and Correlation Analysis of Metrics for Evaluating Performance of Image Fusion, ACTA AUTOMATICA SINICA, Vol. 40(2), pp.:306-315. 